\title{
IEDITORIAL
}

\section{Remote monitoring of cardiovascular implantable electronic devices: a valuable tool for a better care}

Paolo De Filippo, Giovanni Malanchini, Paola Ferrari

Cardiac Electophysiology and Pacing Unit, Ospedale Papa Giovanni XXIII, Bergamo, Italy

\section{RELATED ARTICLE}

by Maciąg et al, see p. 1115
Correspondence to: Giovanni Malanchini, MD, Ospedale Papa Giovanni XXIII, Piazza OMS 1, 24127, Bergamo (BG), Italy, phone: +39035267111 email: gmalanchini@asst-pg23.it Received: October 5, 2020. Accepted: October 6, 2020. Published online: November 25, 2020. Kardiol Pol. 2020; 78 (11): 1086-1087 doi:10.33963/KP.15688 Copyright by the Author(s), 2020
The interest in the clinical and organizational value of remote monitoring of cardiovascular implantable electronic devices (CIEDs) has grown substantially over the last decade. International societies recommend regular follow-up with different timeframes according to different types of CIED..$^{1-3}$ Remote monitoring of CIEDs provides a number of benefits: it allows to adhere to guidelines recommendations and to significantly reduce the amount of time spent in the hospital by patients. It also reduces physician effort for ambulatory consultations and improves patient care by anticipating technical and clinical changes over time. ${ }^{4}$

The role of remote monitoring is even more critical for patients with implantable cardioverter defibrillators (ICDs), both transvenous or subcutaneous, since these patients may benefit the most from rapid interventions. ${ }^{3}$ Main reasons for such interventions are ventricular or supraventricular arrhythmias, lead malfunctioning, or deterioration of clinical status, monitored with indirect indicators such as thoracic impedance and daily activity. .,6 $^{2}$

In this issue of Kardiologia Polska (Kardiol Pol, Polish Heart Journal), Maciąg et $\mathrm{al}^{7}$ address the topic of feasibility of remote monitoring in a large cohort of Polish patients with ICDs and evaluate the effect of such an intervention in an outpatient setting. They followed 176 patients for 12 months and for each patient telemetric visits and outpatient visits were planned in a 3 to 2 ratio. At 1-year follow-up, 91\% of the 352 scheduled outpatient visits and $82 \%$ of the 528 scheduled remote visits were carried out. Moreover, there were 116 (13.4\%) unscheduled visits: 35 (30.2\%) outpatient and 81 (69.8\%) remote visits.
In their study, Maciąg et $\mathrm{al}^{7}$ showed that, during the scheduled visits, episodes of arrhythmias and device malfunctions were detected at similar rates in the outpatient visits and remote visits. Moreover, scheduled remote transmissions, which were performed in line with the study protocol every 3 months, resulted in no intervention in $89.9 \%$ of cases, confirming that the majority of patients do not require routine (every 3-6 month) visits to the outpatient clinic. These findings suggest that remote monitoring could replace the majority of in-person routine follow-up visits.

Conversely, during the unscheduled follow-up visits, both carried out in the office and remotely, arrhythmias were detected significantly more often than in the case of scheduled visits; about a third of unscheduled transmissions required medication interventions, additional consultations, an outpatient visit, or even hospital admission. This reflects one of the greatest advantages of remote monitoring of CIEDs: early detection of adverse events and prompt reaction, leading to major clinical benefits, particularly in patients with device or lead malfunctioning.

Another important issue addressed in the study is that during the study period, an increasing number of patients chose to be remotely monitored and that patient sense of safety increased. In patient opinion, the greatest benefit of the remote monitoring was fast intervention in case of need and an increased sense of safety. Patient acceptance is critical to a successful implementation of remote monitoring in healthcare models. Multiple reasons could account for patient satisfaction with remote monitoring, including ease of use and positive psychological 
impact. Moreover, remote follow-up reduces the social impact of healthcare: less patients have to interrupt their working activities and do not have to travel to reach the hospital.

In conclusion, the study conducted by Maciąg et $\mathrm{al}^{7}$ presents corroborating evidence for the benefits and utility of remote monitoring among patients with CIEDs in a nationwide sample. It describes the feasibility of a postponed in-hospital follow-up, without an increased need for urgent consultations, to confirm good functioning of the implantable cardioverter-defibrillators. Moreover, patients monitored remotely report psychological improvements related to an increased perception of device surveillance. It is reasonable to think that telemedicine will gain more and more space in the future, especially under the revolution caused by the pandemic of coronavirus disease $2019,{ }^{8}$ which imposes a reconstruction of clinical follow-up pathways.

\section{ARTICLE INFORMATION}

DISCLAIMER The opinions expressed by the author are not necessarily those of the journal editors, Polish Cardiac Society, or publisher.

\section{CONFLICT OF INTEREST None declared.}

OPEN ACCESS This is an Open Access article distributed under the terms of the Creative Commons Attribution-NonCommercial-NoDerivatives $4.0 \mathrm{In}$ ternational License (CC BY-NC-ND 4.0), allowing third parties to download articles and share them with others, provided the original work is properly cited, not changed in any way, distributed under the same license, and used for noncommercial purposes only. For commercial use, please contact the journal office at kardiologiapolska@ptkardio.pl.

HOW TO CITE De Filippo P, Malanchini G, Ferrari P. Remote monitoring of cardiovascular implantable electronic devices: a valuable tool for a better care. Kardiol Pol. 2020; 78: 1086-1087. doi:10.33963/KP.15688

\section{REFERENCES}

1 Dubner S, Auricchio A, Steinberg JS, et al. ISHNE/EHRA expert consensus on remote monitoring of cardiovascular implantable electronic devices (CIEDs). Europace. 2012; 14: 278-293.

2 Brunetti ND, Molinari G, Acquistapace F, et al. 2019 Italian Society of Cardiology Census on telemedicine in cardiovascular disease: a report from the working group on telecardiology and informatics. Open Heart. 2020; 7: e001157.

3 De Filippo P, Luzi M, D'Onofrio A, et al. Remote monitoring of subcutaneous implantable cardioverter defibrillators. J Interv Card Electrophysiol. 2018; 53: 373-381.

4 Hindricks G, Elsner C, Piorkowski C, et al. Quarterly vs. yearly clinical follow-up of remotely monitored recipients of prophylactic implantable cardioverter-defibrillators: results of the REFORM trial. Eur Heart J. 2014; 35: 98-105.

5 Malanchini G, Malacrida M, Ferrari P, et al. Impact of the coronavirus disease-19 outbreak on physical activity of patients with implantable cardioverter defibrillators. J Card Fail. 2020; 26: 898-899.

6 Zile MR, Sharma V, Baicu CF, et al. Prediction of heart failure hospitalizations based on the direct measurement of intrathoracic impedance. ESC Heart Fail. 2020; 7: 3040-3048.

7 Maciąg A, Mitkowski P, Mazurek M, et al. Patient perspective and safety of remote monitoring of implantable cardioverter-defibrillators in the Polish Nationwide Multicenter Registry: the Medtronic CareLink network evaluation. Kardiol Pol. 2020; 78: 1115-1121.

8 Varma N, Marrouche NF, Aguinaga L, et al. HRS/EHRA/APHRS/LAHRS/ACC/ AHA worldwide practice update for telehealth and arrhythmia monitoring during and after a pandemic. Heart Rhythm. 2020; 17: e255-e268. 